\title{
Pulsatile Non-Newtonian Laminar Blood Flows through Arterial Double Stenoses
}

\author{
Mir Golam Rabby, Sumaia Parveen Shupti, and Md. Mamun Molla \\ School of Engineering \& Applied Science, Department of Electrical \& Computer Engineering, North South University, \\ Dhaka 1229, Bangladesh \\ Correspondence should be addressed to Md. Mamun Molla; mmamun@northsouth.edu
}

Received 8 March 2014; Accepted 6 May 2014; Published 29 May 2014

Academic Editor: Kuo-Kang Liu

Copyright (C) 2014 Mir Golam Rabby et al. This is an open access article distributed under the Creative Commons Attribution License, which permits unrestricted use, distribution, and reproduction in any medium, provided the original work is properly cited.

\begin{abstract}
The paper presents a numerical investigation of non-Newtonian modeling effects on unsteady periodic flows in a two-dimensional (2D) pipe with two idealized stenoses of $75 \%$ and $50 \%$ degrees, respectively. The governing Navier-Stokes equations have been modified using the Cartesian curvilinear coordinates to handle complex geometries. The investigation has been carried out to characterize four different non-Newtonian constitutive equations of blood, namely, the (i) Carreau, (ii) Cross, (iii) Modified Casson, and (iv) Quemada models. The Newtonian model has also been analyzed to study the physics of fluid and the results are compared with the non-Newtonian viscosity models. The numerical results are represented in terms of streamwise velocity, pressure distribution, and wall shear stress (WSS) as well as the vorticity, streamlines, and vector plots indicating recirculation zones at the poststenotic region. The results of this study demonstrate a lower risk of thrombogenesis at the downstream of stenoses and inadequate blood supply to different organs of human body in the Newtonian model compared to the non-Newtonian ones.
\end{abstract}

\section{Introduction}

Stenosis is characterized by localized arterial narrowing that is initiated due to deposition of lipid, cholesterol, and some other substances on the endothelium and is of major concern to most of the Western world. Atherosclerotic lesions preferentially occur in arteries and arterioles in regions of high curvature or bifurcations and junctions causing major changes in flow structure and consequently large changes in fluid loading on vessel walls [1]. Such plaques or arterial constrictions usually disturb normal blood flow through the artery and there is considerable evidence that hydrodynamic factors can play a significant role in the development and progression of these lesions. It has been established that once a mild stenosis is developed inside the arterial lumen, the resulting flow disorder further influences the development of the disease and the arterial deformability to some extent, which eventually changes the regional blood rheology as well [2].

The rheological behavior of blood can be identified by non-Newtonian viscosity. Halder [3] demonstrated that the rheology of blood and the fluid dynamical properties of blood flow can play an important role in the basic understanding, diagnosis, and treatment of many cardiovascular and arterial diseases. Now, stenosis not only develops in one position of artery but also it may develop at more than one location of the cardiovascular system. However, in many medical situations, the patient is found to have multiple stenoses in the same arterial segment. So, several studies were conducted by Misra et al. [4], Minagar et al. [5], Johnston and Kilpatrick [6], and Mustapha and Amin [7] to understand the effects of double stenoses on blood flow in arteries. A numerical investigation has been conducted for generalized Newtonian blood flows past a couple of irregular arterial stenoses [8].

A few studies have been carried out on multiple stenoses using the momentum integral method and finite element method. There are also many papers devoted to studying these phenomena experimentally. Kilpatrick et al. [9] performed one of the most extensive works on double stenoses where they worked on the vascular resistance of arterial 
stenoses in series. However, Ang and Mazumdar [10] worked on triplet stenoses and their research presented that multiple stenoses have more significant effects on blood flow compared to the sum of the consequences of the individual stenoses. Blood flow through irregular multistenoses has been investigated with and without magnetohydrodynamic effect by Mustapha et al. [11, 12].

Using computed outcomes founded on Galerkin finite element method $\mathrm{Tu}$ et al. [13] executed numerical simulations of models for steady and pulsatile blood flow for distinct constriction levels and Reynolds numbers inside the artery with rigid wall. Talukder et al. [14] experimented the consequences of multiple stenoses on pressure. Young and Tsai [15] experimentally investigated the steady and pulsatile flow aspects through stenotic arteries and found significant pressure decrease across the stenosis. On the contrary, Tu and Deville [16] presented a theoretical analysis of pulsatile flow of blood in stenosed arteries.

Blood is a complex mixture of cells, proteins, lipoproteins, and ions by which nutrients and wastes are transported. Red blood cells typically comprise approximately $40 \%$ of blood by volume. As red blood cells are small semisolid particles, they increase the viscosity of blood and affect the behavior of the fluid. Blood is approximately four times more viscous than water. Moreover, blood does not exhibit a constant viscosity at all flow rates and is especially non-Newtonian in the microcirculatory system [17]. Most of the authors have paid more attention in the investigation of the blood flow by assuming that the blood is Newtonian and homogeneous fluid. However, the non-Newtonian behavior is most evident at very low shear rates when the red blood cells clump together into larger particles. According to Berger and Jou [1] and Huang et al. [18], the shear rates fall below that asymptotic level when the viscosity of blood increases and the non-Newtonian properties of blood are exhibited, especially when the shear rates drop below $10 \mathrm{~s}^{-1}$. Blood also exhibits non-Newtonian behavior in small branches and capillaries, where the cells squeeze through microvasculature and a cell-free skimming layer reduces the effective viscosity through the tube.

The presence of moderate or severe stenoses in the artery can cause the flow to transit from laminar to transition in the downstream region. Moreover, wall pressure and wall shear stress initiated by stenoses play important roles in hemodynamics. Fry [19] revealed that high wall shear stress caused by atherosclerosis is a strong factor for endothelial or inner side damage in an artery. It can again overstimulate platelet thrombosis causing blockage [20]. Therefore, it is important to study the hemodynamic factors to understand the fundamental scenario behind the physiology of arterial diseases.

The aim of the present study is to investigate the nonNewtonian modeling effects on the unsteady periodic flow through an arterial segment with two stenoses of different degree using the most well-documented blood constitutive equations, namely, the Carreau [21], Cross [22], Modified González and Moraga [23], and Quemada [24] models. Newtonian and non-Newtonian flow computations have been carried out elaborately in order to examine the modeling

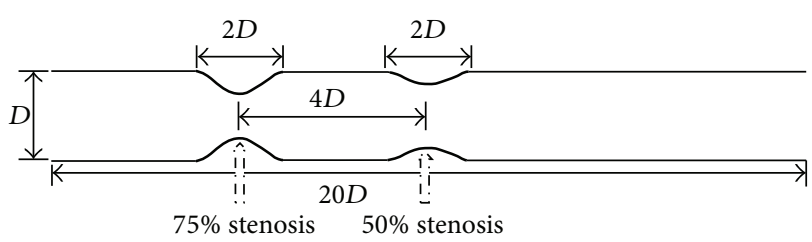

FIGURE 1: Schematic diagram for the double arterial stenoses.

effects with respect to the vortex formation, wall pressure, wall shear stress, and streamlines to achieve very good flow insight into a stenotic artery.

\section{Governing Equation}

Incompressible, homogeneous, and sinusoidal pulsatile flow is simulated for Reynolds number 300. The arterial wall is assumed rigid and blood is modeled as both Newtonian and non-Newtonian fluids for the flow field computation.

The geometry of the two-dimensional (2D) pipe with two cosine-shaped stenoses constricted symmetrically on both sides of the central axis is shown in Figure 1. The artery upstream and downstream to the stenoses have been considered as a straight rigid pipe. Due to the presence of the stenoses, the height of the pipe, $\delta$, is a variable in the streamwise direction (i.e., $\delta=\delta(x)$ ). Away from the stenosis, the height of the pipe is constant and is represented here using $D$ (i.e., $\delta=D$ in the region either upstream or downstream of the stenosis). First stenosis is centered $5 D$ downstream of the pipe inlet (i.e., the inlet location is $x / D=-5$ ) and second stenosis is centered $4 D$ downstream of the first stenosis. The stenoses are centered at $x / D=0.0$ and $x / D=4.0$ axial locations and each stenosis length is $2 D$. The length of the prestenotic region has been considered to be smaller than the poststenotic region for detailed flow insight into the downstream region. The cosine-shaped symmetrically constricted regions are modeled using the following formula:

$$
\frac{y}{D}=1-\frac{f_{c}}{2}\left(1+\cos \frac{x \pi}{D}\right) ; \quad-D \leq x \leq D,
$$

where $f_{c}=(1 / 2)\left[1-((100-\text { percentage }) / 100)^{2}\right]$ is a parameter that controls the reduction of the cross-sectional area of stenosis. In the present study, a $75 \%$ and $50 \%$ reduction of the cross-sectional area has been considered at the center of the first and second stenosis, correspondingly. Generally, the $75 \%$ constricted stenosis is referred to as critical stenosis whereas the $50 \%$ constricted one is referred to as severe stenosis.

Here $x$ and $y$ are used to represent the streamwise and radial coordinates, respectively. Also the tensor notation is 
used in these two directions and it is represented by indices 1 and 2 , respectively:

$$
\begin{gathered}
\frac{\partial u}{\partial x}+\frac{\partial v}{\partial y}=0 \\
\rho\left(\frac{\partial u}{\partial t}+u \frac{\partial u}{\partial x}+v \frac{\partial u}{\partial y}\right) \\
=-\frac{\partial p}{\partial x}+\frac{\partial}{\partial x}\left(2 \mu \frac{\partial u}{\partial x}\right)+\frac{\partial}{\partial y}\left(\mu \frac{\partial u}{\partial y}\right)+\frac{\partial}{\partial y}\left(\mu \frac{\partial u}{\partial x}\right), \\
\rho\left(\frac{\partial v}{\partial t}+u \frac{\partial v}{\partial x}+v \frac{\partial v}{\partial y}\right) \\
=-\frac{\partial p}{\partial y}+\frac{\partial}{\partial x}\left(\mu \frac{\partial v}{\partial x}\right)+\frac{\partial}{\partial y}\left(2 \mu \frac{\partial u}{\partial y}\right)+\frac{\partial}{\partial x}\left(\mu \frac{\partial u}{\partial y}\right) .
\end{gathered}
$$

The blood viscosity, $\mu=\mu(|\dot{\gamma}|)$, depends on the shear rate $\dot{\gamma}=(1 / 2)\left(\left(\partial u_{i} / \partial x_{j}\right)+\left(\partial u_{j} / \partial x_{i}\right)\right)$, and its magnitude is defined as $|\dot{\gamma}|=\sqrt{2 \dot{\gamma}_{i j} \dot{\gamma}_{j i}}$. When blood is treated as a Newtonian fluid, its viscosity tends to a constant value which is denoted by $\mu_{\infty}=3.45 \times 10^{-3} \mathrm{~Pa} \cdot \mathrm{s}$, while for a non-Newtonian model, constitutive relations are used for the apparent viscosity of the blood that are presented in Section 3.

To compute the blood flow though double constricted artery, the governing equations are transformed into curvilinear coordinates. Thompson et al. [25] introduced an approach where the finite difference equations are formulated in a transformed curvilinear coordinate system that coincides with the boundaries of the fluid domain. In this approach flow domain in physical space is mapped onto a rectangular domain in computational space, as shown in Figure 3. For mapping $x_{j} \rightarrow \xi_{j}$, if $J_{i j}$ represents the elements of the Jacobian matrix, $\mathbf{J}$, of the transformation then

$$
J_{i j}=\frac{\partial x_{i}}{\partial \xi_{j}}
$$

The determinate of the Jacobian matrix, $\mathbf{J}$, is denoted by $|\mathbf{J}|$ and given by

$$
|\mathbf{J}|=\frac{\partial x_{i}}{\partial \xi_{j}} A_{i j}
$$

where $A_{i j}$ are the elements of the cofactor matrix, $\mathbf{A}$, of the Jacobian, defined as

$$
|\mathbf{A}|=|\mathbf{J}| \mathbf{J}^{-1} .
$$

By applying the chain rule, the derivatives can now be expressed in the transformed variables in the following way:

$$
\frac{\partial \phi}{\partial x_{i}}=\frac{\partial \phi}{\partial \xi_{j}} \frac{\partial \xi_{j}}{\partial x_{i}}=\frac{A_{i j}}{|J|} \frac{\partial \phi}{\partial \xi_{j}}
$$

where $\phi$ is a generic variable.
The governing equations for an incompressible flow take the following forms in the general Cartesian curvilinear coordinate system:

$$
\begin{aligned}
& \frac{A_{11}}{|\mathbf{J}|} \frac{\partial u}{\partial \xi_{1}}+\frac{A_{12}}{|\mathbf{J}|} \frac{\partial u}{\partial \xi_{2}}+\frac{A_{21}}{|\mathbf{J}|} \frac{\partial v}{\partial \xi_{1}}+\frac{A_{22}}{|\mathbf{J}|} \frac{\partial v}{\partial \xi_{2}}=0, \\
& \rho \frac{\partial u}{\partial t}+\frac{\rho}{|\mathbf{J}|}\left[u A_{11}+v A_{21}\right] \frac{\partial u}{\partial \xi_{1}}+\frac{\rho}{|\mathbf{J}|}\left[u A_{12}+v A_{22}\right] \frac{\partial u}{\partial \xi_{2}} \\
& =-\left[\frac{A_{11}}{|\mathbf{J}|} \frac{\partial p}{\partial \xi_{1}}+\frac{A_{12}}{|\mathbf{J}|} \frac{\partial p}{\partial \xi_{2}}\right]+\frac{1}{\left|\mathbf{J}^{2}\right|} \frac{\partial}{\partial \xi_{1}}\left(\mu \frac{\partial u}{\partial \xi_{1}}\right) \\
& \times\left(2 A_{11}^{2}+A_{21}^{2}\right) \\
& +\frac{1}{\left|\mathbf{J}^{2}\right|} \frac{\partial}{\partial \xi_{1}}\left(\mu \frac{\partial u}{\partial \xi_{2}}\right)\left(2 A_{11} A_{12}+A_{21} A_{22}\right) \\
& +\frac{1}{\left|\mathbf{J}^{2}\right|} \frac{\partial}{\partial \xi_{2}}\left(\mu \frac{\partial u}{\partial \xi_{1}}\right)\left(2 A_{11} A_{12}+A_{21} A_{22}\right) \\
& +\frac{1}{\left|\mathbf{J}^{2}\right|} \frac{\partial}{\partial \xi_{2}}\left(\mu \frac{\partial u}{\partial \xi_{2}}\right)\left(2 A_{12}^{2}+A_{22}^{2}\right) \\
& +\frac{A_{21}}{|\mathbf{J}|} \frac{\partial}{\partial \xi_{1}}\left(\mu\left\{\frac{A_{11}}{|\mathbf{J}|} \frac{\partial v}{\partial \xi_{1}}+\frac{A_{12}}{|\mathbf{J}|} \frac{\partial v}{\partial \xi_{2}}\right\}\right) \\
& +\frac{A_{22}}{|\mathbf{J}|} \frac{\partial}{\partial \xi_{2}}\left(\mu\left\{\frac{A_{11}}{|\mathbf{J}|} \frac{\partial v}{\partial \xi_{1}}+\frac{A_{12}}{|\mathbf{J}|} \frac{\partial v}{\partial \xi_{2}}\right\}\right), \\
& \rho \frac{\partial v}{\partial t}+\frac{\rho}{|\mathbf{J}|}\left[u A_{11}+v A_{21}\right] \frac{\partial v}{\partial \xi_{1}}+\frac{\rho}{|\mathbf{J}|}\left[u A_{12}+v A_{22}\right] \frac{\partial u}{\partial \xi_{2}} \\
& =-\left[\frac{A_{21}}{|\mathbf{J}|} \frac{\partial p}{\partial \xi_{1}}+\frac{A_{22}}{|\mathbf{J}|} \frac{\partial p}{\partial \xi_{2}}\right]+\frac{1}{\left|\mathbf{J}^{2}\right|} \frac{\partial}{\partial \xi_{1}}\left(\mu \frac{\partial v}{\partial \xi_{1}}\right) \\
& \times\left(A_{11}^{2}+2 A_{21}^{2}\right) \\
& +\frac{1}{\left|\mathbf{J}^{2}\right|} \frac{\partial}{\partial \xi_{1}}\left(\mu \frac{\partial v}{\partial \xi_{2}}\right)\left(A_{11} A_{12}+2 A_{21} A_{22}\right) \\
& +\frac{1}{\left|\mathbf{J}^{2}\right|} \frac{\partial}{\partial \xi_{2}}\left(\mu \frac{\partial v}{\partial \xi_{1}}\right)\left(A_{11} A_{12}+2 A_{21} A_{22}\right) \\
& +\frac{1}{\left|\mathbf{J}^{2}\right|} \frac{\partial}{\partial \xi_{2}}\left(\mu \frac{\partial v}{\partial \xi_{2}}\right)\left(A_{12}^{2}+2 A_{22}^{2}\right) \\
& +\frac{A_{21}}{|\mathbf{J}|} \frac{\partial}{\partial \xi_{1}}\left(\mu\left\{\frac{A_{11}}{|\mathbf{J}|} \frac{\partial u}{\partial \xi_{1}}+\frac{A_{12}}{|\mathbf{J}|} \frac{\partial u}{\partial \xi_{2}}\right\}\right) \\
& +\frac{A_{22}}{|\mathbf{J}|} \frac{\partial}{\partial \xi_{2}}\left(\mu\left\{\frac{A_{11}}{|\mathbf{J}|} \frac{\partial u}{\partial \xi_{1}}+\frac{A_{12}}{|\mathbf{J}|} \frac{\partial u}{\partial \xi_{2}}\right\}\right),
\end{aligned}
$$

where $A_{i j}$ are the elements of the cofactor matrix, $\mathbf{A}$, of the Jacobian $|\mathbf{J}|$.

2.1. Boundary Conditions. In the present study, no slip condition has been used at the arterial wall, where velocity $u$ and $v$ are zero. The sinusoidal pulsatile laminar velocity profile $u(r)=2 U\left[1-(y / R)^{2}\right][1+0.3 \sin t]$ is used to generate 


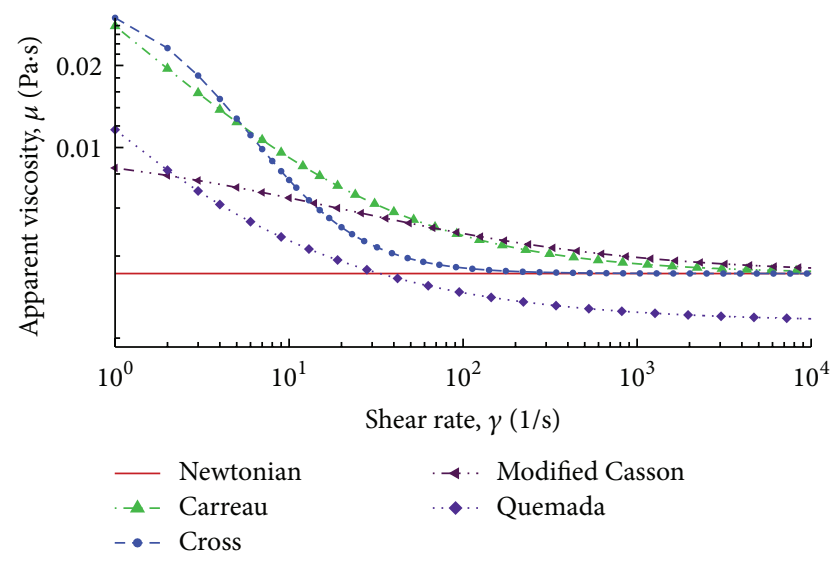

Figure 2: Relations between the shear rate and the apparent blood viscosity for the different models.

the time-dependent pulsatile boundary condition at the inlet of pipe, where bulk velocity, $U$, depends on the Reynolds number and $y$ is the radial length of artery. The zero gradient condition is applied at the outlet of artery, where velocity gradient $u$ and $v$ are zero along the streamwise direction.

\section{Non-Newtonian Viscosity Models}

Newtonian fluid viscosity is always constant against the shear rate. On the other hand, non-Newtonian fluid viscosity changes depending on the shear rate. Figure 2 represents the relationship between blood viscosity and the shear rate for Newtonian and four different non-Newtonian models. The Newtonian and these four non-Newtonian models are summarized in Table 1.

The relationship between the shear rates and viscosity for the non-Newtonian blood viscosity models, that is, Carreau, Cross, Modified Casson, and Quemada models, along with the Newtonian viscosity model is presented in Figure 2. Blood viscosity is constant in the Newtonian model shown by the solid line. On the other hand, the viscosity of blood produced by non-Newtonian models for low shear rates (less than $100 \mathrm{~s}^{-1}$ ) is higher than that of the Newtonian model. Viscosity in the Carreau and Modified Casson models tends to asymptotic constant viscosity, $\mu_{\infty}$, at the shear rate, $\dot{\gamma}$, greater than $10^{4} \mathrm{~s}^{-1}$. The Quemada and the Cross models exhibit the non-Newtonian properties of blood at shear rates rate, $\dot{\gamma}$, less than $10^{4} \mathrm{~s}^{-1}$. Particularly, viscosity asymptotically matches the constant viscosity at the shear rates, $\dot{\gamma}$, greater than $10^{2} \mathrm{~s}^{-1}$ in the Cross model. Quemada model shows the asymptotic nature below the constant viscosity, $\mu_{\infty}$.

\section{Numerical Procedures}

A three-point backward difference formula is used for time derivation of the velocity where the central difference is used for the convective and diffusion terms. Here a pressure correction algorithm is used and pressure as well as the velocity components are stored at the center of a control volume according to the collocated grid arrangement. The
Poisson like pressure correction equation is discretised using the pressure smoothing approach, which prevents the evenodd node uncoupling in the pressure and velocity fields. A BI-CGSTAB [26] solver is used for solving the matrix of velocity vectors, while for the Poisson like pressure correction equation a ICCG [27] solver is applied due to its symmetric and positive definite nature. Overall, the code is second-order accurate in both time and space.

The three-dimensional (3D) version of the present code has been successfully used for various numerical simulation involving LES and DNS techniques. The code is named as BOFFIN (Body Fitted Flow Integrator) which was developed in Imperial College, London, and the details of the program can be found in [28]. It has extensively been used in different 3D pulsatile flow simulations [29-35].

\section{Results and Discussion}

The geometry of arteries has a vital control on blood flow pattern and a local luminal constriction like stenosis greatly disturbs the velocity field. In stenotic flows, of particular interest are the phenomena of the vortex generation and propagation as well as the distribution of the wall shear stress (WSS). According to Neofytou and Drikakis [36], these are considered the most prominent attributes for blood flows because of their relation to atheroma formation in arteries. In line with these two, a detailed description about a set of hemodynamic factors like wall pressure and centerline velocity in streamwise direction with vector plot and vorticity can give a better understanding about the relationship between the fluid dynamics in pulsatile blood flow and arterial disease like stenosis. In this section, plots are demonstrated to show the results of the numerical investigation of blood flow for both Newtonian and non-Newtonian cases through a double stenoses model.

The grid independence test has been carried out to establish a suitable combination of the grid configuration to adequately resolve the flow for different viscous fluid in the stenoses. Fixing the Reynolds number at 300, three computations have been performed for three different grid systems with $150 \times 70$ (Case 1), $180 \times 80$ (Case 2), and $210 \times 90$ (Case 3) control volumes (in the $x$ and $y$ directions, resp.).

The number of streamwise grid points upstream of the first stenosis is always fixed at 30 while the rest of the grid points are distributed nonuniformly within and downstream of the first stenosis. Figure 4 shows the $x-y$ view of a portion of grid system and here it is clearly seen that the grid is significantly refined in order to accurately resolve the wall shear stress in the near-wall region.

The results of Cases 1,2, and 3 are compared in Figure 5 in terms of the nondimensionalized streamwise velocity at different streamwise locations. It is observed from the figure that results for all three cases collapse to almost the same solution throughout the artery having a bit variation in frames 5(i) and (j) which are the poststenotic arterial positions. So, the agreement found in particular for the streamwise velocity is quite good and the flow is well resolved by the grids used in simulation. Therefore, it can be concluded that the present grid is capable of providing convergent solution independent 
TABLE 1: Non-Newtonian models with given molecular viscosity of blood.

\begin{tabular}{|c|c|}
\hline Model & Effective viscosity $^{*}$ \\
\hline Newtonian & $\mu=3.45 \times 10^{-3} \mathrm{~Pa} \cdot \mathrm{s}$ \\
\hline \multirow{4}{*}{ Carreau $\longrightarrow$ Carreau [21] } & $\mu(|\dot{\gamma}|)=\mu_{\infty}+\left(\mu_{0}-\mu_{\infty}\right)\left[1+(\lambda \dot{\gamma})^{2}\right]^{(n-1) / 2}$ \\
\hline & $\mu_{0}=0.056 \mathrm{~Pa} \cdot \mathrm{s}$ viscosity at zero shear rate \\
\hline & $\lambda=3.131$ time constant \\
\hline & $n=0.3568$ \\
\hline \multirow{4}{*}{ Cross $\longrightarrow$ Cross [22] } & $\mu(|\dot{\gamma}|)=\mu_{\infty}+\frac{\left(\mu_{0}-\mu_{\infty}\right)}{\left[1+\left(\dot{\gamma} / \gamma_{c}\right)^{n}\right]}$ \\
\hline & $\mu_{0}=0.0364 \mathrm{~Pa} \cdot \mathrm{s}$, at a very low shear rate \\
\hline & $\gamma_{c}=2.63 \mathrm{~s}^{-1}$ \\
\hline & $n=1.45$ \\
\hline \multirow{5}{*}{ Modified Casson $\longrightarrow$ Gonzalez and Moraga [23] } & $\mu(|\dot{\gamma}|)=\left(\sqrt{\eta_{c}}+\frac{\sqrt{\tau_{0}}}{\sqrt{-}}\right)$ \\
\hline & $\eta_{c}=3.45 \times 10^{-3}$ \\
\hline & $\tau_{0}=2.1 \times 10^{-2} \mathrm{~s}^{-1}$ \\
\hline & $\lambda=11.5 \mathrm{~s}^{-1}$ \\
\hline & $n=1.45$ \\
\hline \multirow{5}{*}{ Quemada $\longrightarrow$ Quemada [24] } & $\mu(|\dot{\gamma}|)=\mu_{p}\left(1-\frac{1}{2} \frac{k_{0}+k_{\mathrm{\infty}} \sqrt{|\dot{\gamma}| / \gamma_{c}}}{1+\sqrt{|\dot{\gamma}| / \gamma_{c}}} \phi\right.$ \\
\hline & $\phi=0.45$ for haematocrit \\
\hline & $\mu_{p}=1.2 \times 10^{-3}$ \\
\hline & $\gamma_{c}=1.88 \mathrm{~s}^{-1}$ \\
\hline & $k_{\infty}=2.07$ and $k_{0}=4.33$ \\
\hline
\end{tabular}

${ }^{*}$ Viscosity is a function of Global shear rate, $|\dot{\gamma}|$ in non-Newtonian models.

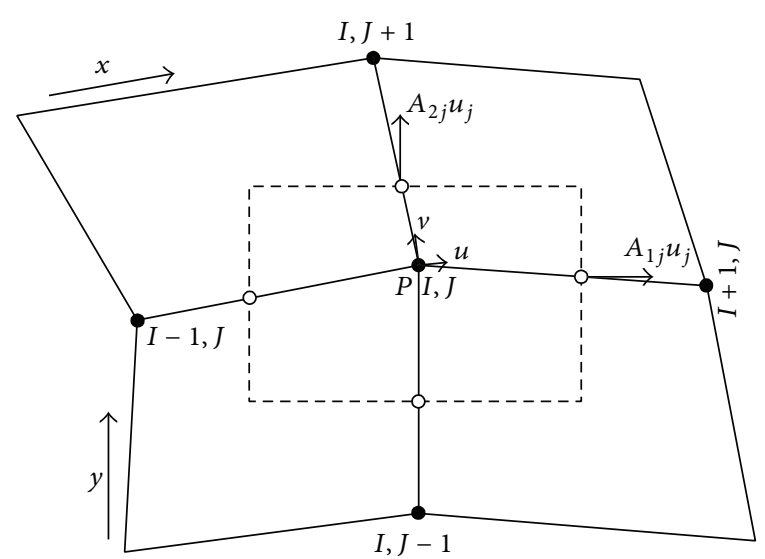

(a)

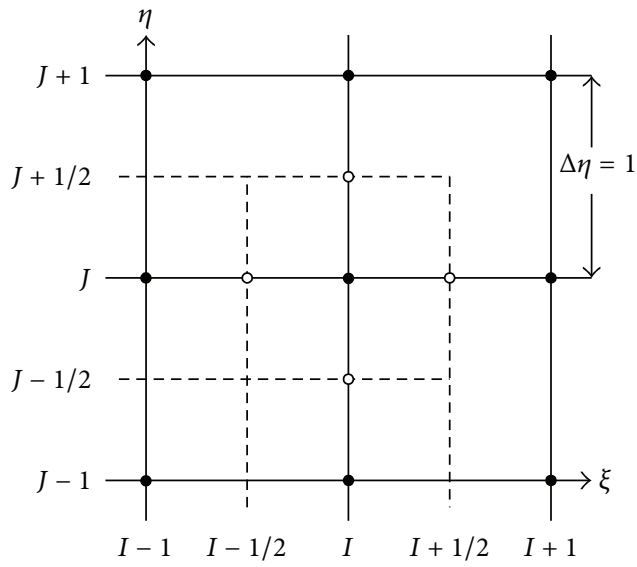

(b)

Figure 3: Grid arrangement and notation in two-dimensional case in both physical space (a) and in computational space (b). Solid lines indicated the grid lines and dashed lines indicated the faces of the control volume.

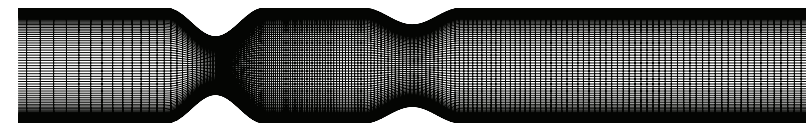

Figure 4: A portion of grid system $(x-y$ view). 


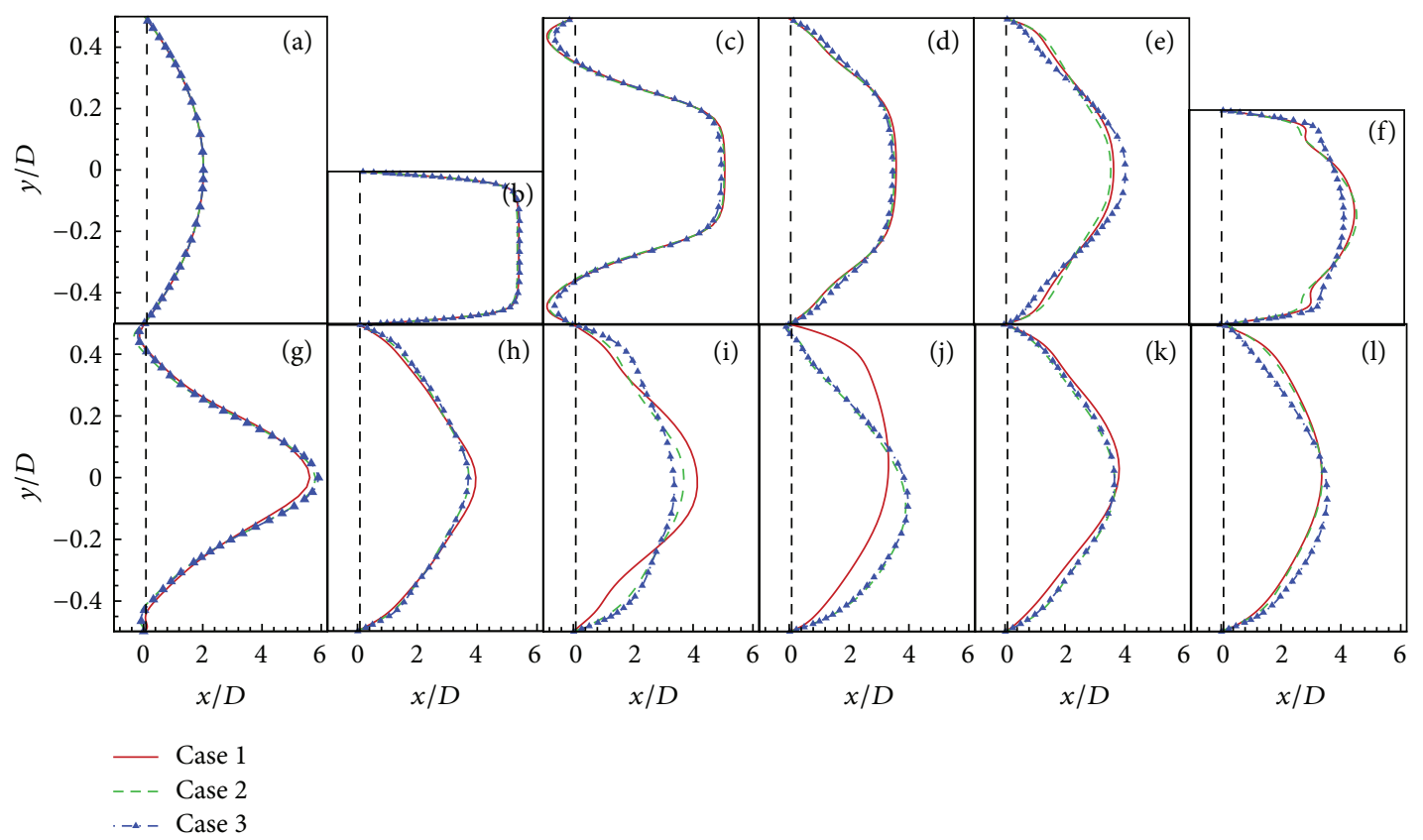

FIGURE 5: Grid independence test with respect to streamwise velocity, $u / U$ at (a) $x / D=-5.0$ (inlet), (b) $x / D=0.0$, (c) $x / D=1.0$, (d) $x / D=2.0$, (e) $x / D=3.0$, (f) $x / D=4.0$, (g) $x / D=5.0$, (h) $x / D=7.0$, (i) $x / D=9.0$, (j) $x / D=11.0$, (k) $x / D=13.0$, and (l) $x / D=15.0$ (outlet). Based on three-grid arrangements, Case 1: solid line for $150 \times 70$ control volumes, Case 2: dashed line for $180 \times 80$ control volumes, and Case 3: line with symbol for $210 \times 90$ control volumes.

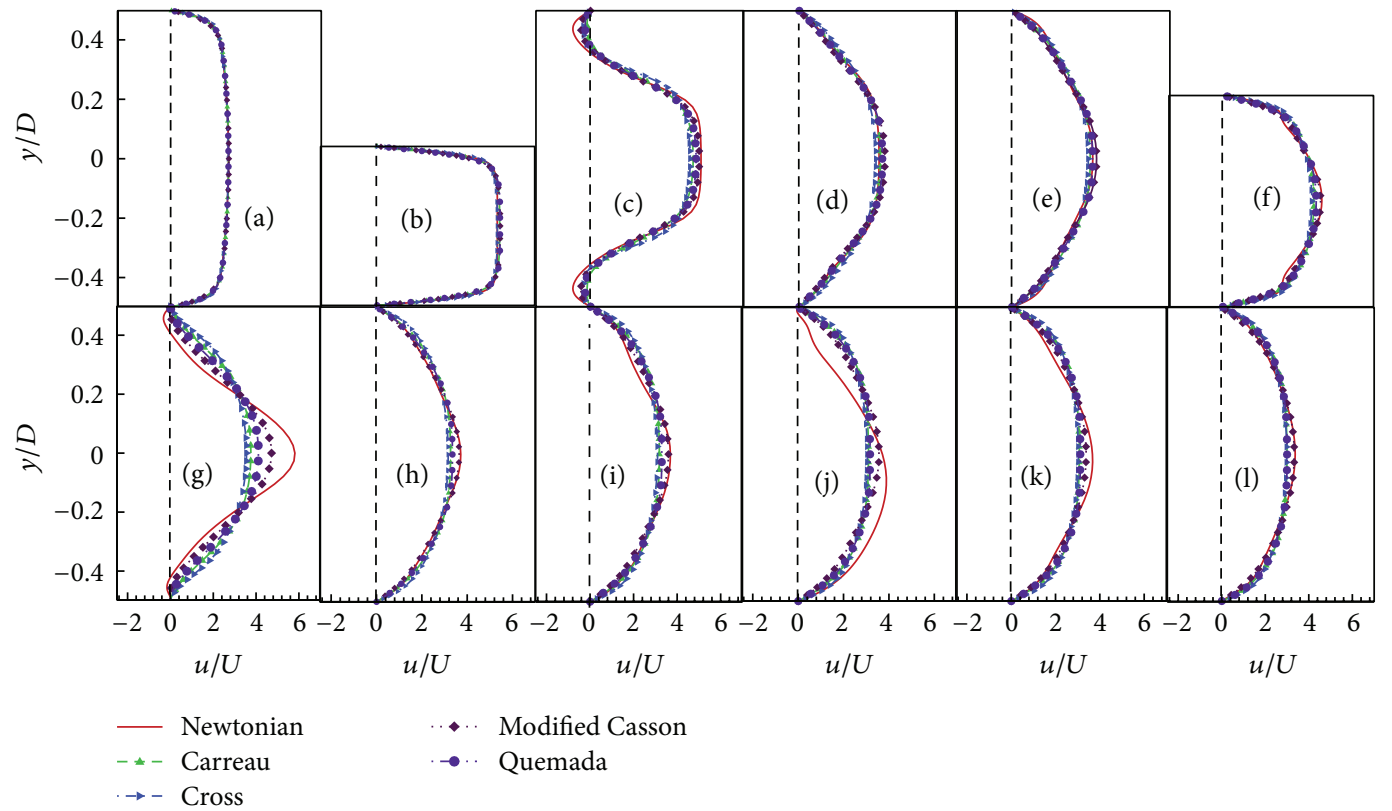

FIGURE 6: Streamwise velocity $u / U$, for different oscillation at the different axial position (a) $x / D=-5.0$ (inlet), (b) $x / D=0.0$, (c) $x / D=1.0$, (d) $x / D=2.0$, (e) $x / D=3.0$, (f) $x / D=4.0$, (g) $x / D=5.0$, (h) $x / D=7.0$, (i) $x / D=9.0$, (j) $x / D=11.0$, (k) $x / D=13.0$, and (l) $x / D=15.0$ (outlet).

of different grid sizes. Based on the satisfactory agreement above the grid arrangement of $180 \times 80$ (Case 2) has been used for all other simulation.

As we have discussed earlier, the presence of stenosis causes a very disturbed flow inside an arterial segment.
Hence, it is important to resolve the magnitude of velocity at every point inside the artery in order to study the pulsatile flow behavior.

The nondimensionalized streamwise velocity $(u / U)$ recorded at different axial locations is presented in 


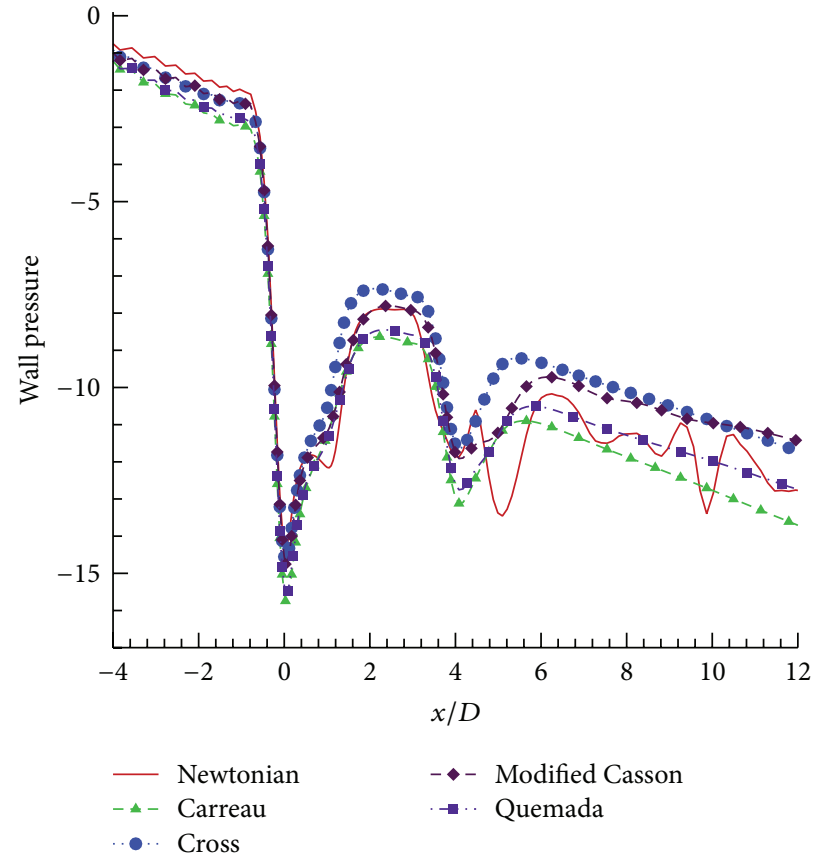

(a)

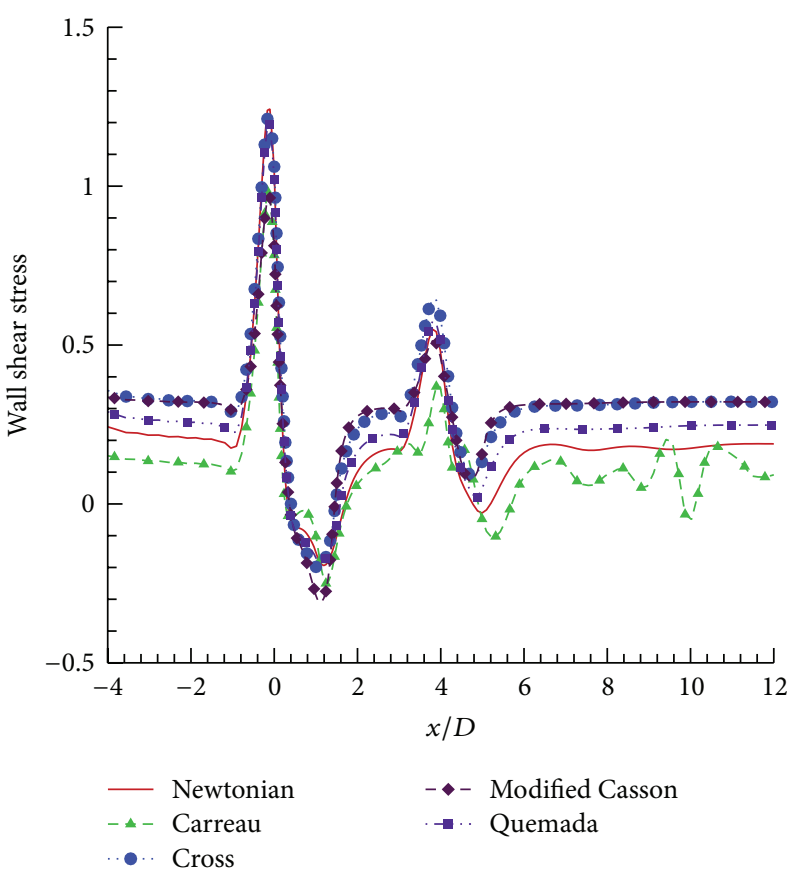

(b)

FIgURE 7: (a) Wall pressure, $\rho / \rho U^{2}$ and (b) wall shear stress, $\tau_{w} / \rho U^{2}$ for different viscosity models, while $\operatorname{Re}=300$.

Figures 6(a)-6(1) for the Newtonian and different nonNewtonian models considering $\mathrm{Re}=300$. The streamwise velocity, whose pattern in the inlet corresponds to sinusoidal laminar pulsatile profile, increases the most with a value of 5.5 at the neck of the critical stenosis shown in frame Figure 6(b). It then decreases in the downstream locations. The velocity again increases in upstream of the severe stenosis and reaches another peak value of 4.5 at the throat location which is shown in frame Figure 6(f). The Newtonian and four other non-Newtonian models show more or less similar velocity profiles up to the neck of the severe stenosis. But their patterns diverse erotically in the downstream location of the severe stenosis shown in frame Figure $6(\mathrm{~g})$. The Newtonian model shows the highest magnitude with Gaussian shaped velocity profile followed by Modified Casson model while with distorted parabolic profile Cross model shows the lowest value followed by Carreau and Quemada models (in increasing order). All five models show almost similar velocity profiles with the some exceptions in the Newtonian one in frames Figures $6(\mathrm{~h})-6(\mathrm{k})$. The velocity decreases and does not change significantly towards the further downstream of the second stenosis since the flow settles down.

Newtonian fluid always maintains constant viscosity and the rate of this viscosity is always lower than the nonNewtonian fluid viscosity. It happens because, after the poststenotic region the velocity of Newtonian fluid is comparatively higher than the non-Newtonian fluid. Negative values of velocity in the downstream location of both stenoses near the upper and lower wall correspond to the presence of permanent recirculation zones shown in frames $6(\mathrm{c})$ and $(\mathrm{g})$.
Again, due to the narrowing of the artery segment caused by stenosis formation, flow gets a slender region inside. According to the Bernoulli equation, the velocity increases the most at the center of both stenoses since the area is smaller therein.

Wall pressure, $p$, normalized by $\rho U^{2}$ for the Newtonian and the non-Newtonian models considering physiological pulsatile inlet flow is shown in Figure 7(a). It is seen that the wall pressure suddenly drops at the neck of the first stenosis having $(x / D=0) 75 \%$ constriction. After this position wall pressure tries to recover but does not attain as higher values as the initial positions. Having another lowest peak at the throat location of the severe stenosis, it follows an oscillating pattern through the downstream regions. The Newtonian and four non-Newtonian models show almost similar pattern where the non-Newtonian models show smooth curves but the Newtonian one shows a bit more zigzag pattern near the downstream of both stenoses. An important observation is that the wall pressure always maintains negative value for all viscous fluids in this rigid wall and double stenosed model artery segment. The diverging-converging shape of the artery seems to have an effect on this pressure distribution.

The blood pressure in most arteries is unsteady and more specifically it is pulsatile in nature which causes variation in pattern of blood pressure during different cardiac phases. The aorta serves as a compliance chamber that provides a reservoir of high pressure during diastole as well as systole and thus blood pressure does not go to zero during diastole even [17]. However, the lowest wall pressure values are found at the throat locations of the stenoses in the present study which are again negative. As a result, the artery might collapse 


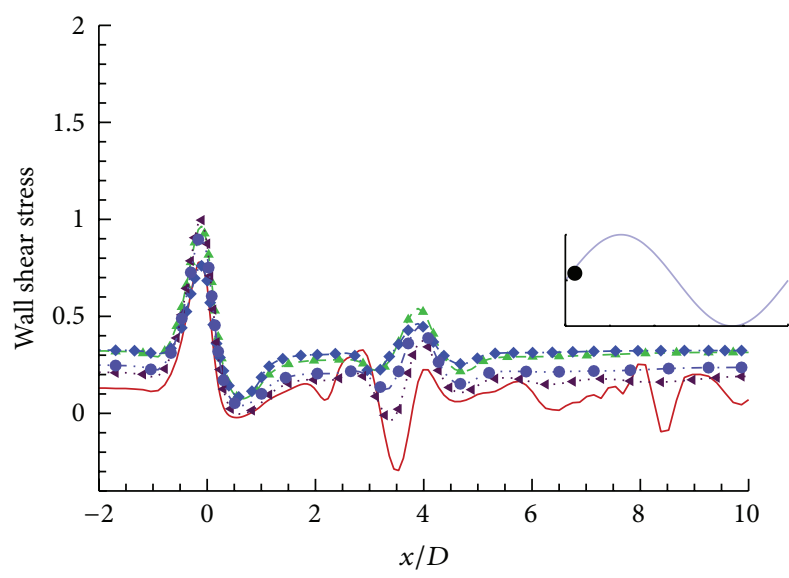

(a)

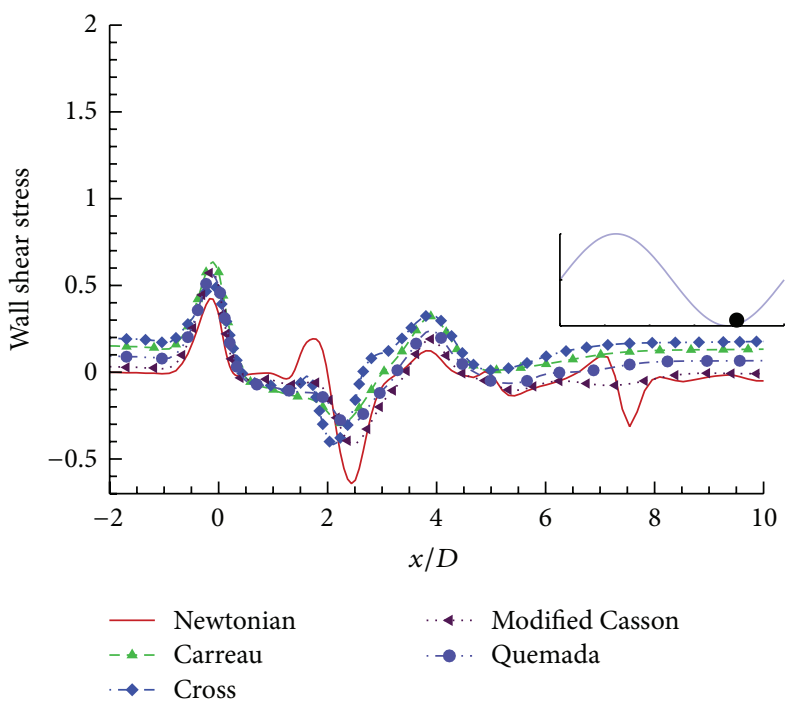

(c)

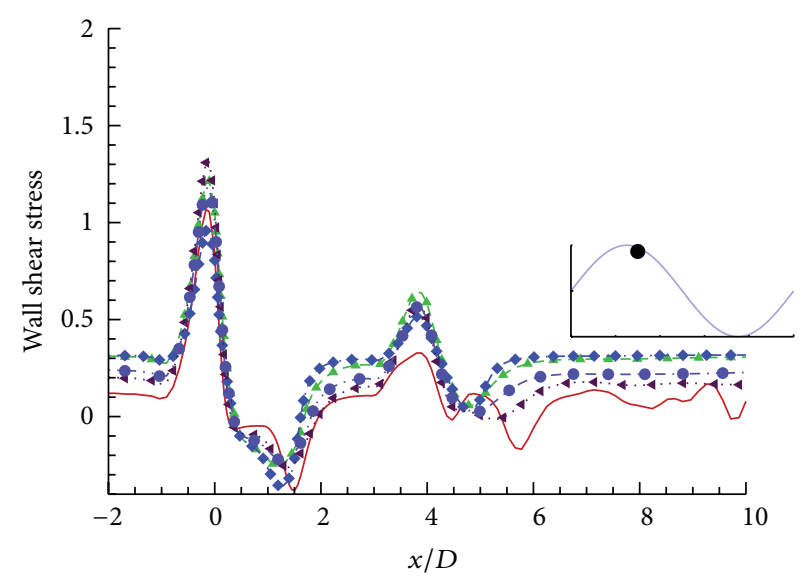

(b)

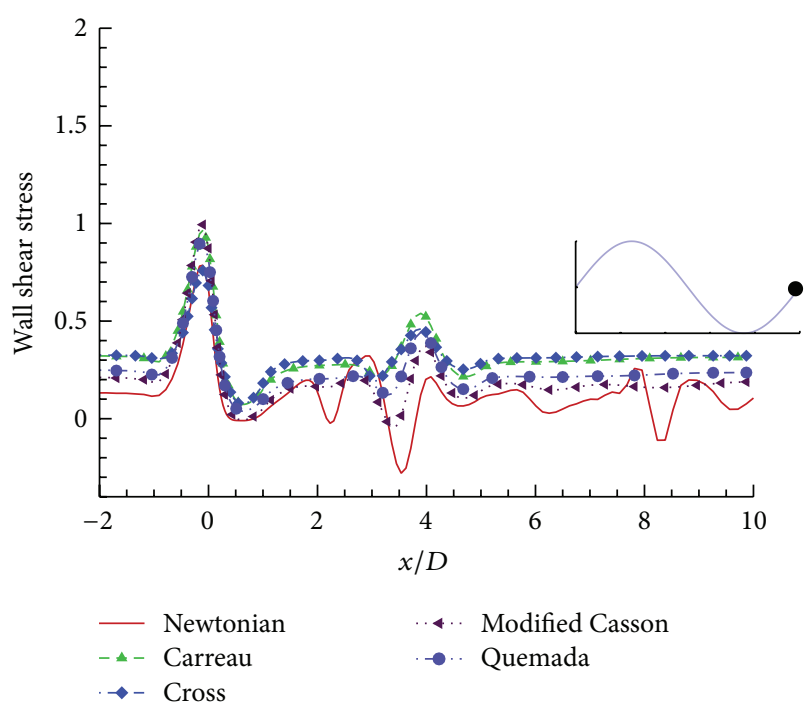

(d)

Figure 8: Wall shear stress, $\tau_{w} / \rho U^{2}$, at different pulsatile phase (a) $t / T=9.0$, (b) $t / T=9.3$, (c) $t / T=9.7$, and (d) $t / T=9.9$, while Re $=300$.

at the neck due to insufficient pressure to maintain the opening of the lumen. Geometric influences on pressure losses have also been studied here and it is found that the pressure drop is so significant in the critical stenosis that the effect of the $50 \%$ stenosis is negligible since the two stenoses are close to one another which matches the results of Seeley and Young [37].

Distribution of the wall shear stress, $\tau_{w}$, normalized by $\rho U^{2}$ caused by the Newtonian and non-Newtonian fluids is depicted in Figure 7(b). Both the Newtonian and nonNewtonian fluids follow the same pattern for wall shear stress in this stenosed artery where the highest peak occurs at the center of the critical stenosis $(x / D=0$ position $)$ and another peak is observed at the center of the severe stenosis $(x / D=4$ position). A similar result was found in case of asymmetric shaped single stenosis model where peak WSS occurred at the throat location by Neofytou and Drikakis [36]. Very high shear stresses near the throat of the stenosis can activate platelets and thereby induce thrombosis, which can totally block blood flow to the heart or brain [17]. The lowest peaks are observed to occur at the distal ends of both stenoses at $x / D=1$ and $x / D=5$ positions, respectively. In the pre- and poststenotic regions, the wall shear stress remains almost constant for all models whereas the Carreau model maintains a very irregular pattern with zigzags and also the minimum magnitudes among all viscosity models including the Newtonian one. Additionally, the Cross and Modified Casson models show comparatively higher wall shear stress throughout the arterial segment than the other three viscosity variant fluids.

According to $\mathrm{Ku}$ [17], the cyclic nature of the heart pump creates pulsatile conditions in all arteries. Moreover, heart always maintains two cyclic phases. It ejects and fills with blood in alternating cycles called systole and diastole. Blood is pumped out of the heart during systole. The heart rests during diastole and no blood is ejected.

The WSS through an arterial segment with critical and severe stenoses for $\operatorname{Re}=300$ considering different phases 


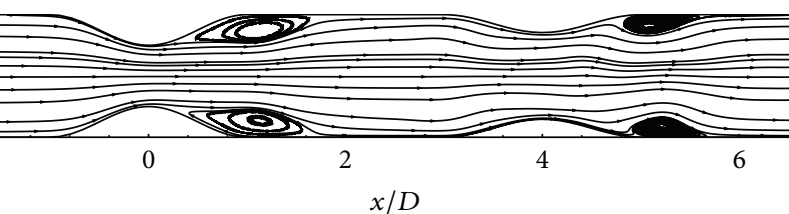

(a)

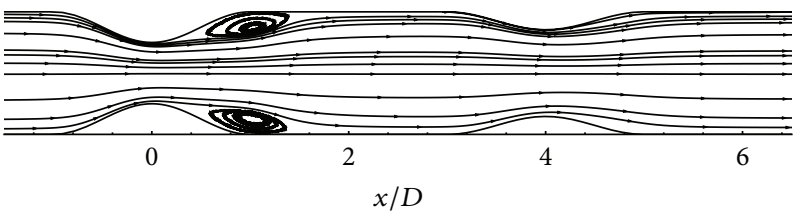

(c)

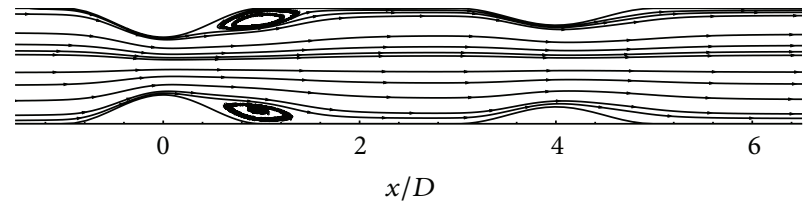

(b)

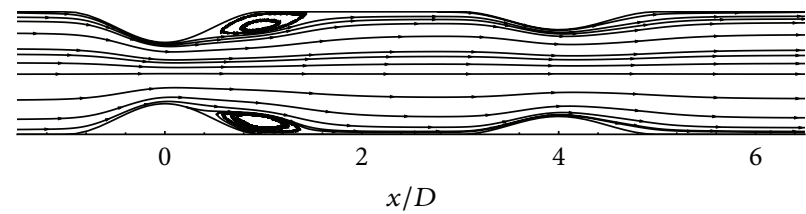

(d)

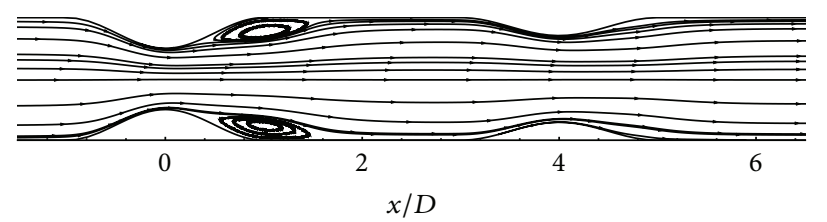

(e)

FIgURE 9: Streamlines for five different viscosity models: (a) Newtonian, (b) Carreau, (c) Cross, (d) Modified Casson, and (e) Quemada models for $\operatorname{Re}=300$.

of a cardiac cycle has been evaluated in this work which is depicted in Figure 8. These WSS diagrams are illustrated based on a sinusoidal cycle which is equivalent to a cardiac cycle. Four distinct phases of a sinusoidal cycle, that is, early systole, peak systole, peak diastole, and late diastole, are plotted here for WSS which is also shown inset. At a glance over the diagram, it is clearly seen that all five viscous fluids follow similar patterns and two peak values of WSS occur at the throats of two stenoses. Variation in values is mostly prominent in the Newtonian model which again maintains the lowest values in all phases among all five models. On the contrary, the Carreau and Cross models show comparatively higher values in different cardiac phases.

An important observation is that the maximum WSS occurs at the peak systole $(t / T=9.3)$ shown in frame $8(\mathrm{~b})$ while the minimal WSS is observed at the peak diastole $(t / T$ $=9.7$ ) shown in frame 8(c) for both Newtonian and nonNewtonian models. Moreover, it is clearly seen that wall shear stress is approximately the same at the beginning and the end of the cycle. This phenomenon proves that our results become steady eventually.

Figure 9 holds the streamlines caused by pulsatile flow in a double stenosed arterial segment for $\mathrm{Re}=300$. Frame of Figure 9(a) represents the streamline for Newtonian case and frames of Figures 9(b) to 9(e) represent the streamlines for four different non-Newtonian viscosity models (Carreau, Cross, Modified Casson, and Quemada, resp.). In the case of Newtonian fluid, the flow is fully developed inside the constriction having higher magnitudes of velocity field in the contour plot which is significant in the throats and downstream regions of both stenoses. The flow is partially developed in the second constriction with a lower magnitude of velocity field. The high velocity region is comparatively smaller in the non-Newtonian viscous models around the critical stenosis and it is almost absent in the severe stenosis. Only Cross model shows a presence of velocity field inside the severe constriction with a magnitude of 4 . However, the extent of the breadth of the recirculation region from the first stenosis and its effects on the flow field downstream of the second stenosis depend on the stenosis spacing ratio, constriction ratio, and the Reynolds number [38].

Downstream of the critical stenosis two-stationary eddies are formed near the upper and lower wall, the sizes of which are different for every model. For the Newtonian case these eddies are the largest in size, while a second pair of smaller eddies in the downstream of severe stenosis is also generated. In the flow field of the non-Newtonian models, eddies downstream of the constriction are smaller than the Newtonian case and the second pair of eddies is absent. The eddies for the Cross model are the largest while the eddy for the Carreau model is the smallest one. The difference in size of the eddy for each of the models can be explained from the fact that - the behavior of the Carreau model is the most viscous followed by the Modified Casson, the Quemada, Cross, and finally the Newtonian model.

In Table 2, comparisons of the point of separation of the shear layer from the nose of the stenoses and its position of reattachment on the wall at the poststenosis region for the different viscous models are given. The separation of the Newtonian model starts early, as point of separation (PS) is recorded at about 0.02671254 which is an upstream location of the nose of the critical stenosis followed by another PS due to the severe stenosis at 4.990938 , while comparatively late separation is predicted by all the non-Newtonian models. The PS of the Cross and the Quemada models is exactly the same which is at 0.03667778 ; separation occurs a bit later by 
TABLE 2: Nonlinear model results.

\begin{tabular}{lcc}
\hline Model & $\begin{array}{c}\text { Point of separation } \\
(\text { PS })\end{array}$ & $\begin{array}{c}\text { Reattachment point } \\
(\mathrm{RA})\end{array}$ \\
\hline Newtonian & $0.267125,4.990938$ & $1.789380,5.774552$ \\
Carreau & 0.401262 & 1.504084 \\
Cross & 0.366777 & 1.504084 \\
Modified & $0.332934,4.779434$ & $1.730357,5.207934$ \\
Casson & 0.366777 & 1.615282 \\
Quemada &
\end{tabular}

the Carreau model. Only the Newtonian and the Modified Casson models cause flow separation in more than one places.

Comparing all the reattachment (RA) points, it is clear that the Carreau model underpredicts the regime of the poststenosis recirculation of blood, while the Newtonian model has an overall maximum prediction of the recirculation regime in case of critical stenosis. For the severe stenosis, the Newtonian model predicts larger recirculation region than the Modified Casson model.

The viscous effects of blood on the development of the flow along the streamwise direction are presented in Figure 10 for $\mathrm{Re}=300$. In this figure, the streamwise velocity vectors are appended on the contours of the streamwise velocity $u / U$. We find that for all viscous models, the primary recirculation region develops near the postlip of the critical stenosis due to the separation of the shear layer from nose of the stenosis. A small recirculation region is observed at the downstream of the severe stenosis in the Newtonian model which is completely absent in case of the non-Newtonian models. Due to these recirculation, Newtonian fluid provides some negative contour values where the contour level ranges from -0.5 to 5.5 . It is also found that non-Newtonian fluid contour values are always positive.

Vorticity describes the local spinning motion of fluid and it can also be said that vorticity highly depends on viscosity. More insight into the flow separation seen in Figure 10 is given through the streamwise vorticity contours, $\omega=(\partial v / \partial x)-(\partial u / \partial y)$, in Figure 11. Figures 11(a) to 11 (e) represent five different viscosity models, that is, the Newtonian, Carreau, Cross, Modified Casson, and Quemada models, respectively. It is noted that a total of 15 unequal contour levels are plotted between their maximum and minimum values, which can be viewed easily through the legend color bar. The vortex units rotated in the clockwise and anticlockwise direction that gives positive and negative values of $\omega$, respectively. The clockwise rotations are represented by the solid lines where the anticlockwise rotations are represented by dashed lines.

Vorticity is very high in low viscosity models. As a result, more vortices are generated from the nose of the stenosis where the flow separation begins in the Newtonian fluid shown in frame of Figure 11(a) where two vortical structures form in the downstream region of the critical stenosis at $x / D=1.0$; one acts in the anticlockwise direction at the upper wall and the other acts in the clockwise direction near lower wall. Both of them interact with each other and then roll up to downstream region. Another pair of vortical structures of clockwise and anticlockwise direction is also present at the downstream of the second stenosis at $x / D=5.0$. It is also evident from the color legend that the maximum magnitude of clockwise vortices lies in the region of $0<x / D<2.0$, $4.0<x / D<6.0$, and $9.0<x / D<10$. An important observation is that the anticlockwise vortex is absent at the location $9.0<x / D<10$ and its strength is very negligible compared to the clockwise ones.

Berger and Jou [1] found that whereas the individual vortex moves at a speed proportional to that of the flow, the front of a train of vorticity, the vorticity wave, propagates at a much higher speed. The reason for the faster propagation speed of the vorticity wave is vortex multiplication, in which a corotating and a contrarotating vortices are generated out of the original vortex. As a result of this vortex multiplication, the extent of the vortex wave in Newtonian fluid grows on an order of magnitude faster than an individual vortex moves.

The vortex structures at the location of $9.0<x / D<10$ are absent in case of all non-Newtonian models. Moreover, the strength of the vortices is also weaker in these models than that of the Newtonian fluid due to more viscosity. Among all of the viscous models, Modified Casson model shows the largest and the Cross model shows the smallest vortex structures.

\section{Conclusion}

Finite volume numerical simulation of unsteady, incompressible, and homogeneous blood flow in two-dimensional rigid models with double constriction has been presented in this paper. Flow field, flow induced wall pressure, and wall shear stress have been compared for Newtonian and nonNewtonian models (Carreau, Cross, Modified Casson, and Quemada) in a rigid pipe with two axisymmetric shaped stenoses of different degree under pulsatile condition for $\mathrm{Re}=$ 300. The maximum shear stresses are observed to occur at the throat locations of the $75 \%$ and $50 \%$ constricted regions which follow a very oscillating manner. The WSS is also characterized by means of a sequence of different flow stages in one period of the cardiac pulse. The highest value of WSS is found at the peak systole while the lowest one occurs during peak diastole.

Pressure loss can be an important reason of stroke or heart attack since it causes inadequate blood supply to the brain, heart, and other organs which is found at the neck of both stenoses. Moreover, Newtonian fluid causes a very disturbed pattern where pressure drop frequently fluctuates between higher and lower values in the downstream of severe stenosis causing more risk of potential heart attack than the non-Newtonian fluids. The wall pressure maintains a negative value throughout the artery segment which might be explained by the geometric influences. Study of the velocity patterns along the streamwise direction shows the peak velocity at the center of each constriction which can be explained by the Bernoulli equation and the one-dimensional continuity equation.

Streamlines demonstrate the presence of recirculation zones in the flow field which is found in the largest scale 


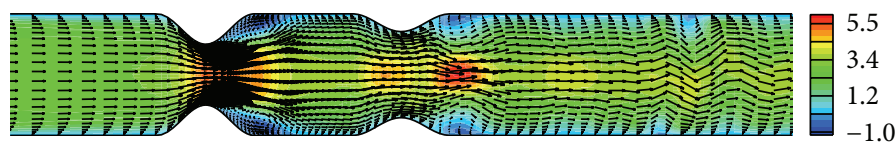

(a)

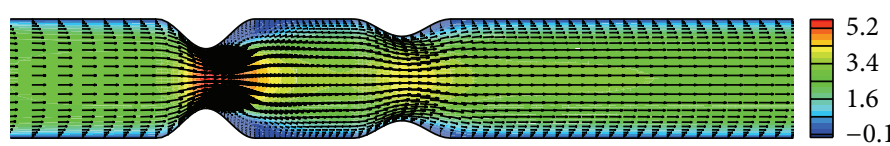

(b)

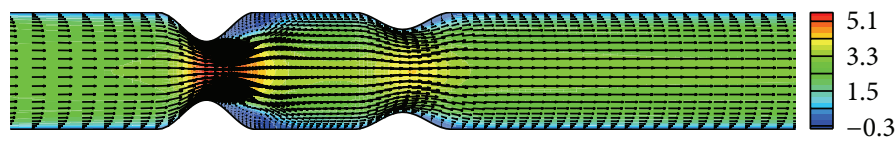

(c)

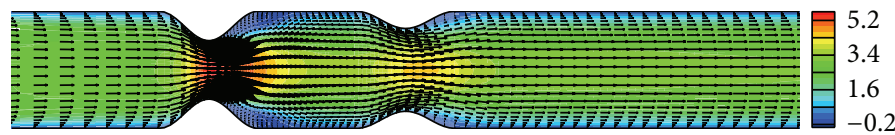

(d)

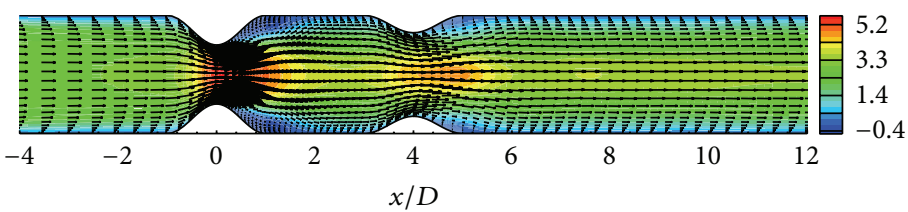

(e)

Figure 10: Vector plot for the different viscosity model appended on the streamwise velocity contour (a) Newtonian, (b) Carreau, (c) Cross, (d) Modified Casson, and (e) Quemada models for $\mathrm{Re}=300$.

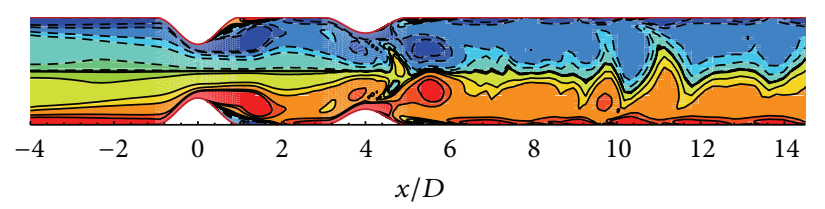

(a)

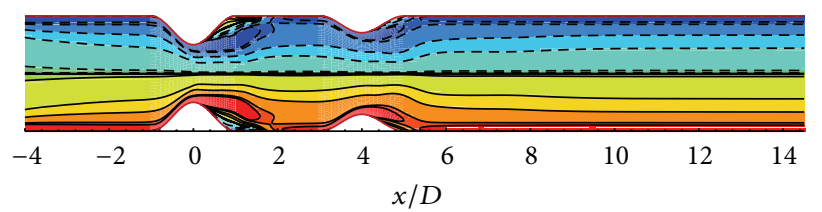

(c)

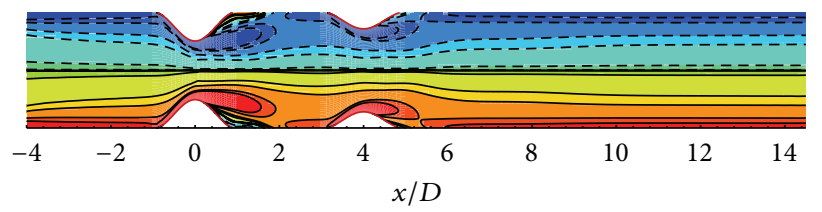

(b)

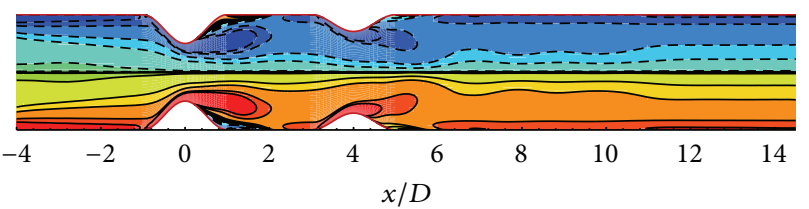

(d)

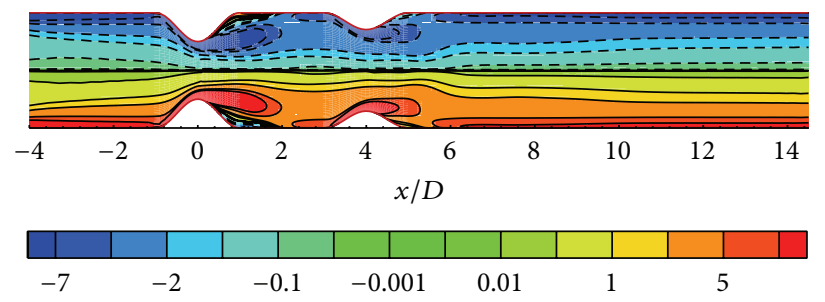

(e)

Figure 11: Vorticity for different viscosity models: (a) Newtonian, (b) Carreau, (c) Cross, (d) Modified Casson, and (e) Quemada models for $\mathrm{Re}=300$. 
by the Newtonian fluid where the flow is fully developed inside both constricted regions. This again increases the possibility of thrombosis. However, the smallest recirculation region is caused by the Carreau model. A very interesting phenomenon of vorticity is observed while investigating its characteristics; the strength of clockwise vortices is higher than the anticlockwise ones. Moreover, the speed or propagation of a vortex train is higher than an individual vortex which is observed in case of Newtonian fluids due to low viscosity.

In conclusion, it can be stated that the Newtonian fluid is more likely to cause heart attack or blockage due to its characteristics of high wall shear stress, pressure loss, and the largest recirculation region at the throat locations of the stenoses than the non-Newtonian models. Limitations of this investigation include the consideration of rigid wall and simple sinusoidal pulsatile inlet profile instead of compliant arterial wall and physiological realistic inlet profile.

\section{Nomenclature}

English Symbols

A: Amplitude of the wall oscillation (m)

$A_{i j}$ : Elements of the cofactor matrix

$D:$ Diameter of artery $(\mathrm{m})$

J: Jacobian

p: $\quad$ Pressure $(\mathrm{Pa})$

$r$ : Radius of the pipe $(\mathrm{m})$

Re: Reynolds number (UD/v)

$t$ : Time (s)

$U:$ Bulk velocity $\left(\mathrm{m} \cdot \mathrm{s}^{-1}\right)$

$u$ : Velocity along the streamwise direction $\left(\mathrm{m} \cdot \mathrm{s}^{-1}\right)$

$v$ : Velocity along the radial direction $\left(\mathrm{m} \cdot \mathrm{s}^{-1}\right)$.

\section{Greek Symbols}

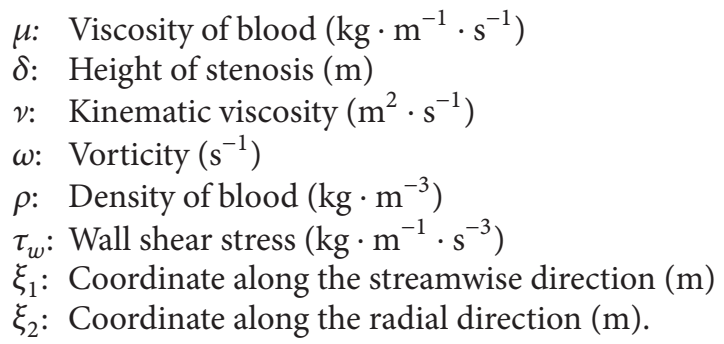

\section{Conflict of Interests}

The authors declare that there is no conflict of interests regarding the publication of this paper.

\section{Acknowledgment}

Mir Golam Rabby and Sumaia Parveen Shupti wish to acknowledge gratefully the funding from the North South University, Bangladesh, during the period of this research.

\section{References}

[1] S. A. Berger and L.-D. Jou, "Flows in stenotic vessels," Annual Review of Fluid Mechanics, vol. 32, pp. 347-382, 2000.

[2] N. Mustapha, S. Chakravarty, P. K. Mandal, and N. Amin, "Unsteady response of blood flow through a couple of irregular arterial constrictions to body acceleration," Journal of Mechanics in Medicine and Biology, vol. 8, no. 3, pp. 395-420, 2008.

[3] K. Haldar, "Effects of the shape of stenosis on the resistance of blood flow through an artery," Bulletin of Mathematical Biology, vol. 47, no. 4, pp. 545-550, 1985.

[4] J. C. Misra, A. Sinha, and G. C. Shit, "Mathematical modeling of blood flow in a porous vessel having double stenoses in the presence of an external magnetic field," International Journal of Biomathematics, vol. 4, no. 2, pp. 207-225, 2011.

[5] A. Minagar, W. Jy, J. J. Jimenez, and J. S. Alexander, "Multiple sclerosis as a vascular disease," Neurological Research, vol. 28, no. 3, pp. 230-235, 2006.

[6] P. R. Johnston and D. Kilpatrick, "Mathematical modelling of paired arterial stenoses," in Proceedings of the Computers in Cardiology, pp. 229-232, September 1990.

[7] N. Mustapha and N. Amin, "The unsteady power law blood flow through a multiirregular stenosed artery," Matematika, vol. 24, no. 2, pp. 189-200, 2008.

[8] N. Mustapha, P. K. Mandal, I. Abdullah, N. Namin, and T. Hayat, "Numerical simulation of generalized newtonian blood flow past a couple of irregular arterial stenoses," Numerical Methods for Partial Differential Equations, vol. 27, no. 4, pp. 960-981, 2011.

[9] D. Kilpatrick, S. D. Webber, and J.-P. Colle, "The vascular resistance of arterial stenoses in series," Angiology, vol. 41, no. 4, pp. 278-285, 1990.

[10] K. C. Ang and J. Mazumdar, "Mathematical modelling of triple arterial stenoses," Australasian Physical and Engineering Sciences in Medicine, vol. 18, no. 2, pp. 89-94, 1995.

[11] N. Mustapha, N. Amin, S. Chakravarty, and P. K. Mandal, "Unsteady magnetohydrodynamic blood flow through irregular multi-stenosed arteries," Computers in Biology and Medicine, vol. 39, no. 10, pp. 896-906, 2009.

[12] N. Mustapha, P. K. Mandal, P. R. Johnston, and N. Amin, "A numerical simulation of unsteady blood flow through multiirregular arterial stenoses," Applied Mathematical Modelling, vol. 34, no. 6, pp. 1559-1573, 2010.

[13] C. Tu, M. Deville, L. Dheur, and L. Vanderschuren, "Finite element simulation of pulse tile flow through arterial stenosis," Journal of Biomechanics, vol. 25, no. 10, pp. 1141-1152, 1992.

[14] N. Talukder, P. E. Karayannacos, R. M. Nerem, and J. S. Vasko, "An experimental study of fluid mechanics of arterial stenosis," Journal of Biomechanical Engineering, vol. 99, no. 2, pp. 74-82, 1977.

[15] D. F. Young and F. Y. Tsai, "Flow characteristics in models of arterial stenoses: I. Steady flow, Journal of Biomechanics, vol. 6, no. 4, pp. 395-410, 1973.

[16] C. Tu and M. Deville, "Pulsatile flow of non-newtonian fluids through arterial stenoses," Journal of Biomechanics, vol. 29, no. 7, pp. 899-908, 1996.

[17] D. N. Ku, "Blood flow in arteries," Annual Review of Fluid Mechanics, vol. 29, no. 1, pp. 399-434, 1997.

[18] C. R. Huang, W. D. Pan, H. Q. Chen, and A. L. Copley, "Thixotropic properties of whole blood from healthy human subjects," Biorheology, vol. 24, no. 6, pp. 795-801, 1987. 
[19] D. L. Fry, "Acute vascular endothelial changes associated with increased blood velocity gradients," Circulation Research, vol. 22, no. 2, pp. 165-197, 1968.

[20] J. D. Folts, E. B. Crowell Jr., and G. G. Rowe, "Platelet aggregation in partially obstructed vessels and its elimination with aspirin," Circulation, vol. 54, no. 3, pp. 365-370, 1976.

[21] P. J. Carreau, "Rheological equations from molecular network theories," Journal of Rheology, vol. 16, no. 1, pp. 99-127, 1972.

[22] M. M. Cross, "Rheology of non-Newtonian fluids: a new flow equation for pseudoplastic systems," Journal of Colloid Science, vol. 20, no. 5, pp. 417-437, 1965.

[23] H. A. González and N. O. Moraga, "On predicting unsteady non-newtonian blood flow," Applied Mathematics and Computation, vol. 170, no. 2, pp. 909-923, 2005.

[24] D. Quemada, "Rheology of concentrated disperse systems III. General features of the proposed non-newtonian model. Comparison with experimental data," Rheologica Acta, vol. 17, no. 6, pp. 643-653, 1978.

[25] J. F. Thompson, F. C. Thames, and C. W. Mastin, "Automatic numerical generation of body-fitted curvilinear coordinate system for field containing any number of arbitrary twodimensional bodies," Journal of Computational Physics, vol. 15, no. 3, pp. 299-319, 1974.

[26] D. S. Kershaw, "The incomplete Cholesky-conjugate gradient method for the iterative solution of systems of linear equations," Journal of Computational Physics, vol. 26, no. 1, pp. 43-65, 1978.

[27] H. A. D. Vorst, "Bi-cgstab: a first and smoothly converging variant of bi-cg for the solution of the non-symmetric linear systems," SIAM Journal on Scientific and Statistical Computing, vol. 155, pp. 631-644, 1992.

[28] W. P. Jones, F. di Mare, and A. J. Marquis, LES-BOFFIN: Users Guide, Mechanical Engineering Department, Imperial College London, London, UK, 2002.

[29] M. C. Paul, M. Mamun Molla, and G. Roditi, "Large-Eddy simulation of pulsatile blood flow," Medical Engineering and Physics, vol. 31, no. 1, pp. 153-159, 2009.

[30] M. C. Paul and M. Mamun Molla, "Investigation of physiological pulsatile flow in a model arterial stenosis using largeeddy and direct numerical simulations," Applied Mathematical Modelling, vol. 36, no. 9, pp. 4393-4413, 2012.

[31] M. Mamun Molla, M. C. Paul, and G. Roditi, "Physiological flow in a model of arterial stenosis," Journal of Biomechanics, vol. 41, supplement 1, p. S243, 2008.

[32] M. Mamun Molla and M. C. Paul, "LES of non-newtonian physiological blood flow in a model of arterial stenosis," Medical Engineering \& Physics, vol. 34, no. 8, pp. 1079-1087, 2012.

[33] M. Mamun Molla, M. C. Paul, and G. Roditi, "LES of additive and non-additive pulsatile flows in a model arterial stenosis," Computer Methods in Biomechanics and Biomedical Engineering, vol. 13, no. 1, pp. 105-120, 2010.

[34] M. Mamun Molla, B. C. Wang, and D. C. Kuhn, "Numerical study of pulsatile channel flows undergoing transition triggered by a modelled stenosis," Physics of Fluids, vol. 24, no. 12, Article ID 121901, 2012.

[35] M. Mamun Molla, A. Hossain, B. C. Wang, and D. C. Kuhn, "Large-eddy simulation of pulsatile non-newtonian flow in a constricted channel," Progress in Computational Fluid Dynamics, vol. 12, no. 4, pp. 231-242, 2012.

[36] P. Neofytou and D. Drikakis, "Effects of blood models on flows through a stenosis," International Journal for Numerical Methods in Fluids, vol. 43, no. 6-7, pp. 597-635, 2003.
[37] B. D. Seeley and D. F. Young, "Effect of geometry on pressure losses across models of arterial stenoses," Journal of Biomechanics, vol. 9, no. 7, pp. 439-448, 1976.

[38] T. S. Lee, W. Liao, and H. T. Low, "Numerical simulation of turbulent flow through series stenoses," International Journal for Numerical Methods in Fluids, vol. 42, no. 7, pp. 717-740, 2003. 

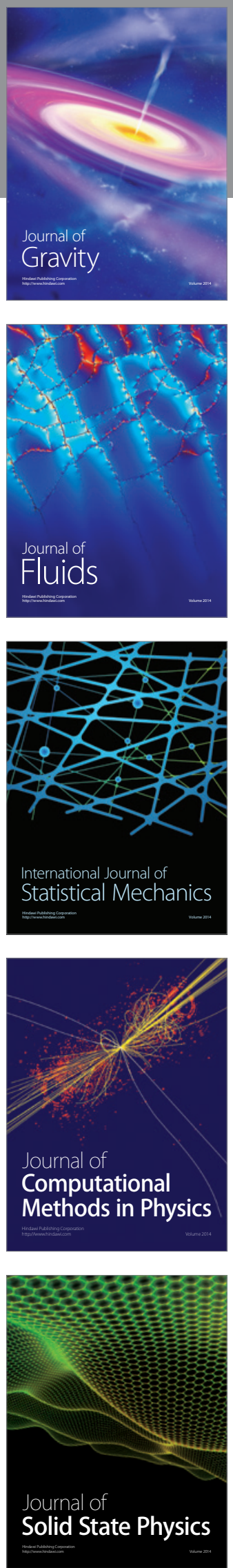

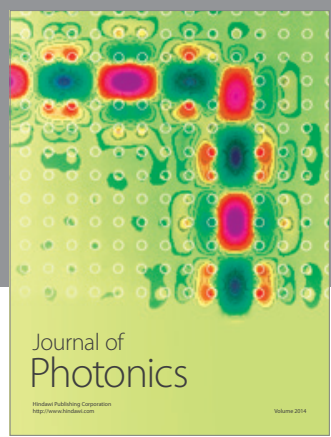

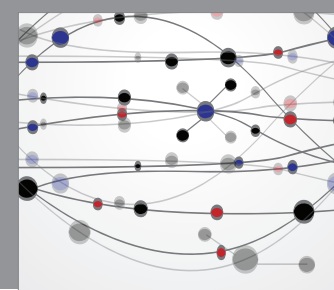

The Scientific World Journal

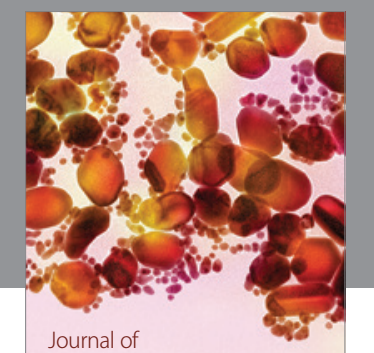

Soft Matter
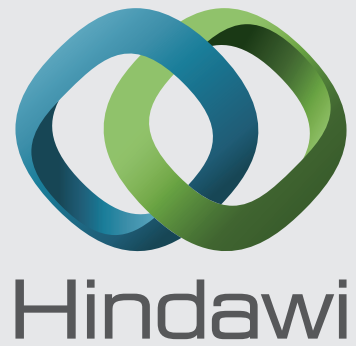

Submit your manuscripts at

http://www.hindawi.com
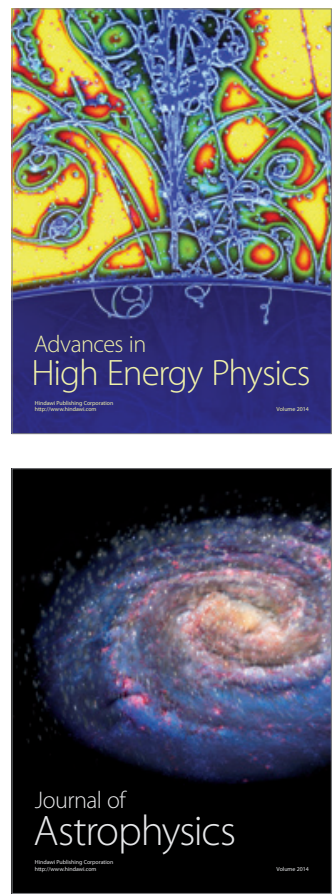
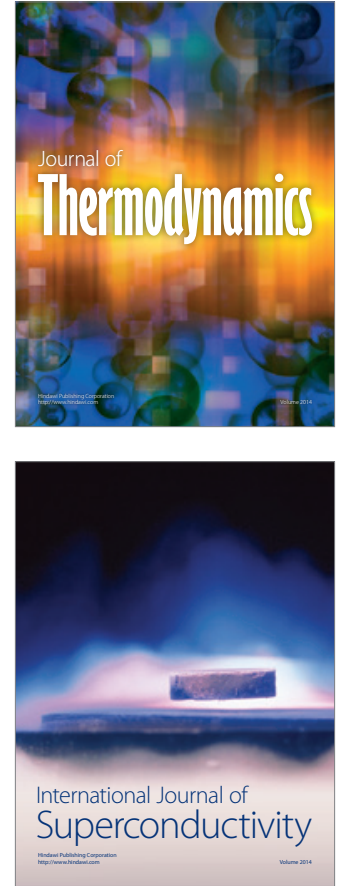
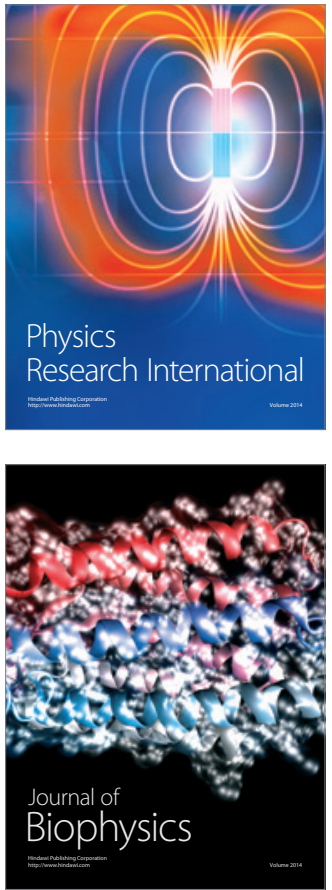
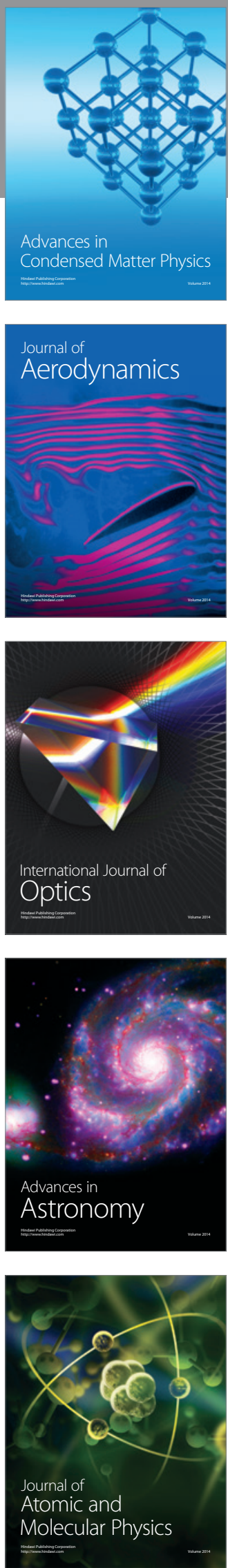